\title{
The influence of mesh removal during laparoscopic repair of recurrent ventral hernias on the long-term outcome
}

\author{
Hasan Ediz Sikar, Kenan Çetin \\ Department of General Surgery, Dr. Lutfi Kirdar Kartal Training and Research Hospital, Istanbul, Turkey
}

Videosurgery Miniinv 2019; 14 (3): 366-373

DOI: https://doi.org/10.5114/wiitm.2019.85350

\begin{abstract}
Introduction: Recurrent hernias can be treated by laparoscopy without the need for mesh removal. However, shrinkage of the mesh following recurrence affects most of the patients, and leaving a partially floating and rigid foreign body could be discussed.

Aim: To compare the outcomes of patients regarding the removal of previous mesh during laparoscopic repair of recurrent abdominal wall hernias.

Material and methods: Patients who underwent laparoscopic repair for recurrent ventral hernias between August 2012 and March 2015 were included in the study. The patients with complete removal of the mesh were included in the MR group and the patients with partial removal or without removal of the previous mesh were included in the non-MR group. Patient characteristics and demographics, previous hernia repair, defect size, mesh size, operative time, mean hospital stay, complications, recurrences, numeric pain rating scale (NRS), early termination of analgesics and prolonged use of analgesics were compared.

Results: A total of 112 patients with a mean age of 53.2 and mean body mass index of $31.1 \mathrm{~kg} / \mathrm{m}^{2}$ underwent laparoscopic repair. There were 47 patients in the MR group and 54 patients in the non-MR group. Operative time was shorter in the non-MR group ( $p<0.05$ ), whereas symptomatic seroma and NRS scores on postoperative day 10 and at the $6^{\text {th }}$ week were higher in comparison with the MR group $(p<0.05)$.

Conclusions: Mesh removal during laparoscopic repair of recurrent ventral hernias has an association with the reduction of pain and symptomatic seroma. However, further prospective comparative studies are required to verify this view.
\end{abstract}

Key words: laparoscopy, incisional hernia, surgical mesh, recurrent hernia, mesh removal, long-term outcome.

\section{Introduction}

Recurrence following abdominal wall hernia repair remains a significant problem [1-6]. Failure of previous repair attempts could be the starting point of a vicious circle. The high recurrence rate reported in the literature indicates the need for standardization regarding the repair methods. Laparoscopic repair allows a decreased recurrence rate and morbidity for incisional hernias [3-6]. Although numerous methods could be used for recurrent hernias, most of the patients have the previous repair with mesh either laparoscopic or open approach. Shrinkage of the mesh associated with scar tissue formation on the synthetic scaffold may lead to recurrence, which may additionally increase this process and mesh deformation. Recurrent hernias can be treated by laparoscopy without the need for mesh removal [2]. However, shrinkage of the mesh affects most of the patients, and leaving a partially floating and rigid

\section{Address for correspondence}

Hasan Ediz Sikar MD, Department of General Surgery, Dr. Lutfi Kirdar Kartal Training and Research Hospital, Istanbul, Turkey,

phone: +90 216 4583000, e-mail: hasan.sikar@me.com 
foreign body has not been comprehensively studied in the literature. Considering the removal of mesh, most of the studies concern mesh infections [7-12]. Data on the efficacy of mesh removal during laparoscopic repair remain missing. The missing data in the literature led us to evaluate whether there is an advantage or disadvantage of mesh removal during a laparoscopic repair.

\section{Aim}

We aimed to compare the outcomes of patients regarding the removal of the previous mesh during laparoscopic repair of recurrent abdominal wall hernias.

\section{Material and methods}

Patients who underwent laparoscopic abdominal wall hernia repair due to recurrence between August 2012 and March 2015 were included in the study. Patients who had a hernia with midline localization were included in the study - except subxiphoid localization. Patients who did not undergo mesh repair previously, who had a contraindication to use dexketoprofen trometamol (DT), who did not have a minimum follow-up of 48 months and were lost to follow-up were excluded. Excluded patients are depicted in Figure 1. Our hospital ethics committee approved the study (Reg. number: 5141502, 2019). Following the division of the branches in the general surgery department, we made a flow-chart to evaluate our experience and to follow up the patients who underwent surgery, in the division of hernia. The medical records were reviewed retrospectively from our department's database. The data were collected prospectively with a follow-up form including patient characteristics and demographics, number of previous hernia repairs, number of previous incisional hernia repairs, defect size, mesh size, operative time, mean hospital stay, complications, recurrences, follow-up time, numeric pain rating scale (NRS), early termination of analgesics and prolonged use of analgesics. All operations were performed by two surgeons experienced in hernia repair in the division of hernia surgery.

The patients were placed in the supine position with the arm of the opposite side tucked. An orogastric tube was inserted routinely following anesthesia induction. An open approach was preferred for optical trocar insertion, and trocars were placed as far as possible from the defect. Three trocars were used to form a triangle, and an additional trocar was placed in the case of difficulty during fixation. Mesh removal was performed following the adhesiolysis. Excision of the mesh and fixation materials from the abdominal wall was done by sharp dissection with the help of electrocautery and vessel sealing devices. The patients with complete removal of the mesh were included in the MR group, and the patients with partial removal or without removal of the previous mesh were included in the non-MR group. The previous mesh was taken out of the abdomen through the optical port incision. Laparoscopic intraperitoneal onlay mesh repair (IPOM) with bridging was performed. The new mesh (Parietene Composite PPC Mesh, Covidien, Mansfield MA, USA) was placed with the help of 4 transfascial sutures and with an adequate overlap of $5 \mathrm{~cm}$ over the defect. The new mesh was placed with the help of 3 transfascial sutures in the case of suprapubic hernia, and the transfascial suture was not applied on the pubis. Titanium helical tacks (Protack, Covidien, Mansfield MA, USA) were used to fix the mesh with the double-crown technique.

Intravenous infusion of dexketoprofen trometamol (DT) was administered $50 \mathrm{mg}$ every $12 \mathrm{~h}$ on postoperative day 1 and in the case of complaints on the following days until discharge. An oral form of DT $25 \mathrm{mg}$ was prescribed on discharge. The patients were examined at the first week, $10^{\text {th }}$ day, sixth week, third month, sixth month and annually to evaluate complaints and pain. In accordance with the follow-up form, the patients were asked whether

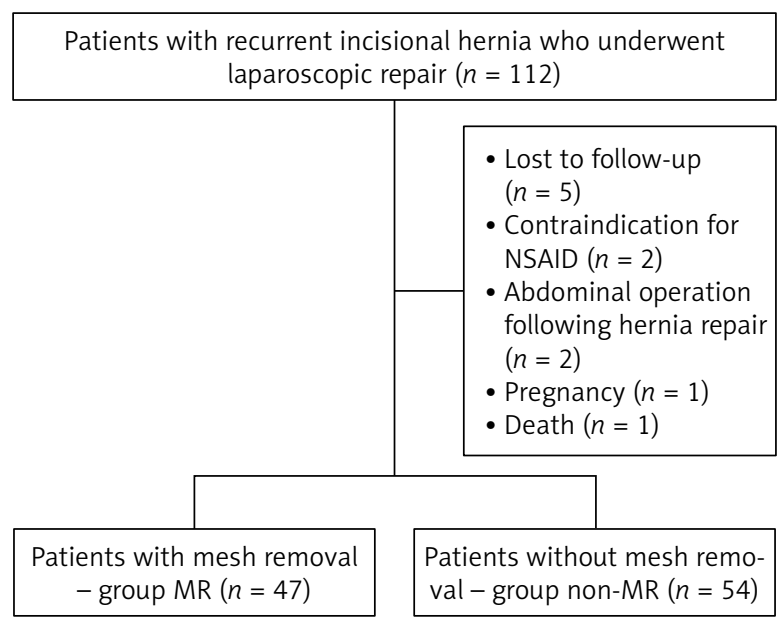

Figure 1. Flow diagram of patients with recurrent ventral and incisional hernias 
they had stopped the use of analgesics in the postoperative first week. In addition, the patients were asked whether they needed the prescription of DT on postoperative day 10 . The patients who had left DT before the first week were grouped as "early termination of analgesics" and patients who had required the prescription of DT on the $10^{\text {th }}$ day were grouped as "prolonged use of analgesics". Patients who required additional analgesics during the postoperative first week or wanted to use another drug for pain on the postoperative $10^{\text {th }}$ day were included in the "prolonged use of analgesics". A numeric pain rating scale with 11 points was applied to evaluate the pain on postoperative days 1 and 10 and the sixth week. The patients were asked to rate pain ranging from " 0 " representing "no pain" to " 10 " representing "the worst possible pain". The patients were asked whether they had a complaint of pain that restricted daily activities at the postoperative third month, sixth month and annually to evaluate chronic pain.

Lower scores of NRS and decreased prolonged use of analgesics in the MR group and long-lasting complaints regarding the pain in the non-MR group needed to be evaluated following the comparison of the groups. There was a requirement of a control group to evaluate the similarity regarding the absence of previous mesh. Therefore, the data of the patients with incisional hernia repair for the first time - who had an occurrence of incisional hernia following laparotomy, without a recurrent hernia at the same interval (August 2012 and March 2015) were compared with the MR group and the non-MR group. Physical examination was done by the same surgeons in the same unit during all visits. In addition, ultrasonography (USG) was used to evaluate the recurrence annually and in the case of suspicion. Seroma was followed until postoperative 8 weeks and absence of regression after 8 weeks was considered to be a chronic seroma. The patients with chronic seroma were evaluated with USG to decide aspiration at the eighth and $10^{\text {th }}$ weeks.

\section{Statistical analysis}

Statistical analysis was carried out using IBM SPSS Statistics ver. 25.0 (IBM Co., Armonk, NY, USA). Descriptive statistical methods (mean, standard deviation, frequency, percent) were used to consider the study data. The quantitative variables were com- pared with independent samples t-test. Pearson's $\chi^{2}$ test and Fisher's exact test were performed to compare qualitative data. Based on the result of the analyses, a $p<0.05$ was considered to be significant.

\section{Results}

A total of 112 patients with recurrent incisional hernia underwent laparoscopic repair between August 2012 and March 2015. Eleven patients were excluded from the study due to various reasons and are depicted in Figure 1. Therefore 101 patients with a mean age of $53.2 \pm 8.8$ and mean body mass index (BMI) of $31.1 \pm 5.4 \mathrm{~kg} / \mathrm{m}^{2}$ were included in the study. The MR group consisted of 47 patients whereas there were 54 patients in the non-MR group. Most of the hernias occurred following gynecological operations - a total of 41 (40.6\%) patients, 21 (20.8\%) in the MR group and 20 in the non-MR group (19.8\%). Thirty-eight (37.6\%) patients had multiple incisions. There was no statistically significant difference between groups regarding previous operations and incisions. Distribution of previous surgery and incisions is depicted in Table I. There was no statistically significant difference between groups regarding age, gender, body mass index (BMI), comorbid disease, smoking, defect size, mesh size, multiple defects, number of previous hernia repairs, number of previous incisional hernia repairs, follow-up time, mean hospital stay and recurrence. Operation time was significantly longer in the MR group in comparison with the non-MR group - 93.34 \pm 26.15 min vs. $78.11 \pm 26.58 \min (p=0.005)$. Comparison of the groups according to the mesh removal is shown in Table II.

The most common complication was chronic seroma, and it affected 9 (8.9\%) patients in the MR group and the non-MR group. Patients with chronic seroma in the non-MR group were more numerous than the MR group, and the difference is statistically significant $-8(14.8 \%)$ patients in the nonMR group and 1 (2.1\%) patient in the MR group $(p<0.05)$. Aspiration was sufficient for patients with chronic seroma, and no additional surgical intervention was required. Single aspiration was sufficient for the patient in the MR group, whereas there were $2(3.7 \%)$ patients who required aspiration three times, 5 (9.3\%) patients who required aspiration two times and 1 (1.8\%) patient who required aspiration one time in the non-MR group. There was no significant difference regarding the NRS postop- 
Table I. Comparison of groups regarding previous incisions and operations that cause incisional hernia

\begin{tabular}{|lccccc|}
\hline $\begin{array}{l}\text { Variable (MR group/ } \\
\text { non-MR group) }\end{array}$ & Gynecological & Bowel & Solid organs & Hernia & Total \\
\hline Upper midline incision & $-/-$ & $2 / 3$ & $2 / 2$ & $2 / 2$ & $6 / 7(p=0.976)$ \\
\hline Paraumbilical incision & $-/-$ & $-/-$ & $-/-$ & $3 / 4$ & $3 / 4(p=1.0)$ \\
\hline Lower midline incision & $8 / 9$ & $3 / 4$ & $-/-$ & $-/-$ & $11 / 13(p=0.937)$ \\
\hline Pfannenstiel incision & $9 / 7$ & $-/-$ & $-/-$ & $-/-$ & $9 / 7(p=0.396)$ \\
\hline Multiple incisions & $4 / 4$ & $11 / 12$ & $2 / 1$ & $1 / 3$ & $18 / 20(p=0.896)$ \\
\hline Total & $21 / 20(p=0.435)$ & $16 / 19(p=0.904)$ & $4 / 3(p=0.702)$ & $6 / 9(p=0.582)$ & $47 / 51$ \\
\hline
\end{tabular}

Table II. Comparison of groups according to mesh removal and incisional hernia repair for the first time

\begin{tabular}{|c|c|c|c|c|c|}
\hline Parameter & $\begin{array}{l}\text { MR group } \\
(n=47)\end{array}$ & $\begin{array}{l}\text { Non-MR group } \\
\quad(n=54)\end{array}$ & $P$-value ${ }^{1}$ & $\begin{array}{l}\text { Primary } \\
\text { incisional } \\
(n=91)\end{array}$ & $P$-value ${ }^{2}$ \\
\hline Age & $51.95 \pm 9.04$ & $54.33 \pm 8.63$ & 0.18 & $49.02 \pm 9.73$ & $0.088 / 0.001$ \\
\hline Gender (female/male) & $\begin{array}{c}39(83.8 \%) / \\
8(17.0 \%)\end{array}$ & $\begin{array}{c}49(90.7 \%) / \\
5(9.3 \%)\end{array}$ & 0.245 & $\begin{array}{l}67(73.6 \%) / \\
24(26.4 \%)\end{array}$ & $0.217 / 0.013$ \\
\hline $\mathrm{BMI}\left[\mathrm{kg} / \mathrm{m}^{2}\right]$ & $31.32 \pm 5.59$ & $30.89 \pm 5.32$ & 0.691 & $29.8 \pm 4.42$ & $0.084 / 0.188$ \\
\hline Comorbid disease & $19(40.4 \%)$ & $27(50 \%)$ & 0.335 & $38(41.8 \%)$ & $0.88 / 0.335$ \\
\hline Smoking & $9(19.1 \%)$ & $10(18.5 \%)$ & 0.936 & $18(19.8 \%)$ & $0.929 / 0.852$ \\
\hline Defect size $\left[\mathrm{cm}^{2}\right]$ & $73.76 \pm 38.23$ & $86.62 \pm 47.0$ & 0.133 & $69.54 \pm 33.3$ & $0.5 / 0.021$ \\
\hline Mesh size $\left[\mathrm{cm}^{2}\right]$ & $402.12 \pm 187.94$ & $469.44 \pm 204.06$ & 0.089 & $385.71 \pm 136.27$ & $0.55 / 0.009$ \\
\hline Multiple defects & $10(21.3 \%)$ & $16(29.6 \%)$ & 0.338 & $20(22 \%)$ & $0.925 / 0.303$ \\
\hline Number of previous hernia repairs & $2.8 \pm 1.45$ & $3.2 \pm 1.75$ & 0.224 & - & - \\
\hline $\begin{array}{l}\text { Number of previous incisional } \\
\text { hernia repairs }\end{array}$ & $1.57 \pm 1.41$ & $1.81 \pm 1.66$ & 0.439 & - & - \\
\hline Operation time [min] & $93.34 \pm 26.15$ & $78.11 \pm 26.58$ & 0.005 & $68.45 \pm 20.05$ & $<0.001 / 0.023$ \\
\hline Mean hospital stay [day] & $1.68 \pm 0.66$ & $1.62 \pm 0.75$ & 0.721 & $1.42 \pm 0.71$ & $0.047 / 0.113$ \\
\hline Recurrence & $3(6.4 \%)$ & $3(5.6 \%)$ & 1.0 & $2(2.2 \%)$ & $0.337 / 0.268$ \\
\hline Follow-up time [month] & $69.17 \pm 8.76$ & $66.55 \pm 8.19$ & 0.125 & $66.73 \pm 8.03$ & $0.1 / 0.897$ \\
\hline NRS postoperative day 1 & $6.14 \pm 0.9$ & $6.05 \pm 1.21$ & 0.667 & $5.85 \pm 0.86$ & $0.067 / 0.297$ \\
\hline NRS postoperative day 10 & $2.91 \pm 0.9$ & $3.31 \pm 0.9$ & 0.029 & $2.78 \pm 0.82$ & $0.382 /<0.001$ \\
\hline NRS postoperative $6^{\text {th }}$ week & $0.21 \pm 0.46$ & $0.57 \pm 0.66$ & 0.002 & $0.16 \pm 0.37$ & $0.512 /<0.001$ \\
\hline Pain restricting activity $3^{\text {rd }}$ month & - & $2(3.7 \%)$ & 0.497 & - & $-/ 0.137$ \\
\hline Pain restricting activity $6^{\text {th }}$ month & - & $1(1.9 \%)$ & 1.0 & - & $-/ 0.372$ \\
\hline Pain restricting activity first year & - & - & - & - & $-1-$ \\
\hline Early termination of analgesics & $14(29.8 \%)$ & $12(22.2 \%)$ & 0.386 & $38(41.8 \%)$ & $0.17 / 0.017$ \\
\hline Prolonged use of analgesics & $5(10.6 \%)$ & $18(33.3 \%)$ & 0.007 & $4(4.4 \%)$ & $0.27 /<0.001$ \\
\hline Chronic seroma & $1(2.1 \%)$ & $8(14.8 \%)$ & 0.035 & $2(2.2 \%)$ & $1.0 / 0.006$ \\
\hline Prolonged ileus & $1(2.1 \%)$ & $2(3.7 \%)$ & 1.0 & $1(1.1 \%)$ & $1.0 / 0.312$ \\
\hline
\end{tabular}

${ }^{1} M R$ group vs. non-MR group, ${ }^{2} M R$ group vs. primary incisional/non-MR group vs. primary incisional. 
erative day 1. NRS of the patients in the MR group was non-significantly higher in comparison with the non-MR group -6.14 vs. 6.05 . The NRS scores were decreased in both groups on the following days (Table II). However, the decrease in the non-MR group was limited. NRS scores were higher in the non-MR group on postoperative day 10 and at the sixth week $(p<0.05)$. There were $2(3.7 \%)$ patients with pain restricting activity on the third month in the nonMR group, whereas there was no patient in the MR group. There was no patient with chronic pain and also pain restricting activity, at the first year and the following check-ups. In addition, prolonged use of analgesics was more frequent in the non-MR group ( $p<0.05)$. Recurrence was observed at postoperative months 26, 39, 51 in the MR group, and at postoperative months $31,49,54$ in the non-MR group. Recurrence was observed in the patients who had a relatively large defect width. The width of the defect ranged from 8 to $11 \mathrm{~cm}$ in the MR group, and also the range of width was between 9 to $13 \mathrm{~cm}$ in the non-MR group.

Comparison of the MR group and the patients with a primary incisional hernia is depicted in Table II. Age and BMI were higher in the MR group, but the difference was not statistically significant - 51.95 and $31.32 \mathrm{~kg} / \mathrm{m}^{2}$ in the MR group vs. 49.02 and $29.8 \mathrm{~kg} / \mathrm{m}^{2}$ in primary incisional patients, respectively. There was no statistically significant difference in terms of gender, defect size, mesh size, recurrence, follow-up time, pain restricting activity in the third month or complications. Operation time and mean hospital stay were significantly shorter in patients with incisional hernia repair for the first time $-68.45 \mathrm{~min}$ vs. $93.34 \min (p<0.001)$ and 1.42 day vs. 1.68 day $(p<0.05)$, respectively. Considering the pain, NRS was non-significantly lower on postoperative days 1 and 10 and at the sixth week in comparison between the two groups with the absence of previous mesh. There was no significant difference regarding early termination of analgesics and prolonged use of analgesics $-29.8 \%$ and $10.6 \%$ in the MR group, $41.8 \%$ and $4.4 \%$ in primary incisional patients, respectively. In addition, the non-MR group and the patients with primary incisional hernia patients were compared to evaluate pain. The difference was significant in terms of NRS on postoperative day 10, at the sixth week, early termination of analgesics, prolonged use of analgesics, operation time, and chronic seroma $(p<0.05)$. However, the groups showed different pa- tient characteristics considering age, gender, mesh size and defect size $(p<0.05)$.

\section{Discussion}

Recurrent abdominal wall hernias lead to the problem of the previous mesh inside. Considering the guideline recommendations, incisional hernia recurrence could be treated without the need for mesh removal [2]. However, depending on the localization and fixation of the previous mesh, the problem could range from an asymptomatic patient to a patient with a solid mass and pain. Regarding mesh removal, most of the studies concern the removal of infected mesh [7-12]. Although some studies concern laparoscopic repair of recurrent ventral hernias, there is still a lack of data about mesh removal during the procedure [13]. Determining the borders of the defect, which consists of an edge with a floating mesh, could be impossible. In addition, considering the open approach, extended skin, subcutaneous tissue, and the mesh are excised for most of the recurrent ventral hernias during surgery. Therefore, the outcome following mesh removal and the technical details should be discussed regarding the laparoscopic repair. Laparoscopic ventral hernia repair provides a better outcome regarding operative time and postoperative infections [3-6]. Removal of the mesh could convert this simple procedure to a complex modality. However, a rigid mass with a partially floating part inside the abdominal cavity, during laparoscopic hernia repair, led us to remove these foreign bodies (Photo 1). We preferred to remove the previous mesh, without a function to support the defect and which could be accepted as a foreign body, in our series. Some of the cases were not suitable for mesh removal: recurrent defects with total bulging of the mesh and adherent or closer to the skin, which had the requirement of skin excision. Considering the possibilities of previous surgery, most of the patients in the MR group had a previous repair of IPOM or sublay methods, whereas most of the patients in the non-MR group had a previous repair of onlay methods.

As an expected finding the operative time was higher in the MR group, due to an additional part of surgery during the excision of mesh and fixation materials $(p<0.05)$. However, operative time did not affect the mean hospital stay, and it was similar for both groups. There was no statistically significant difference between the MR group and the non-MR group 

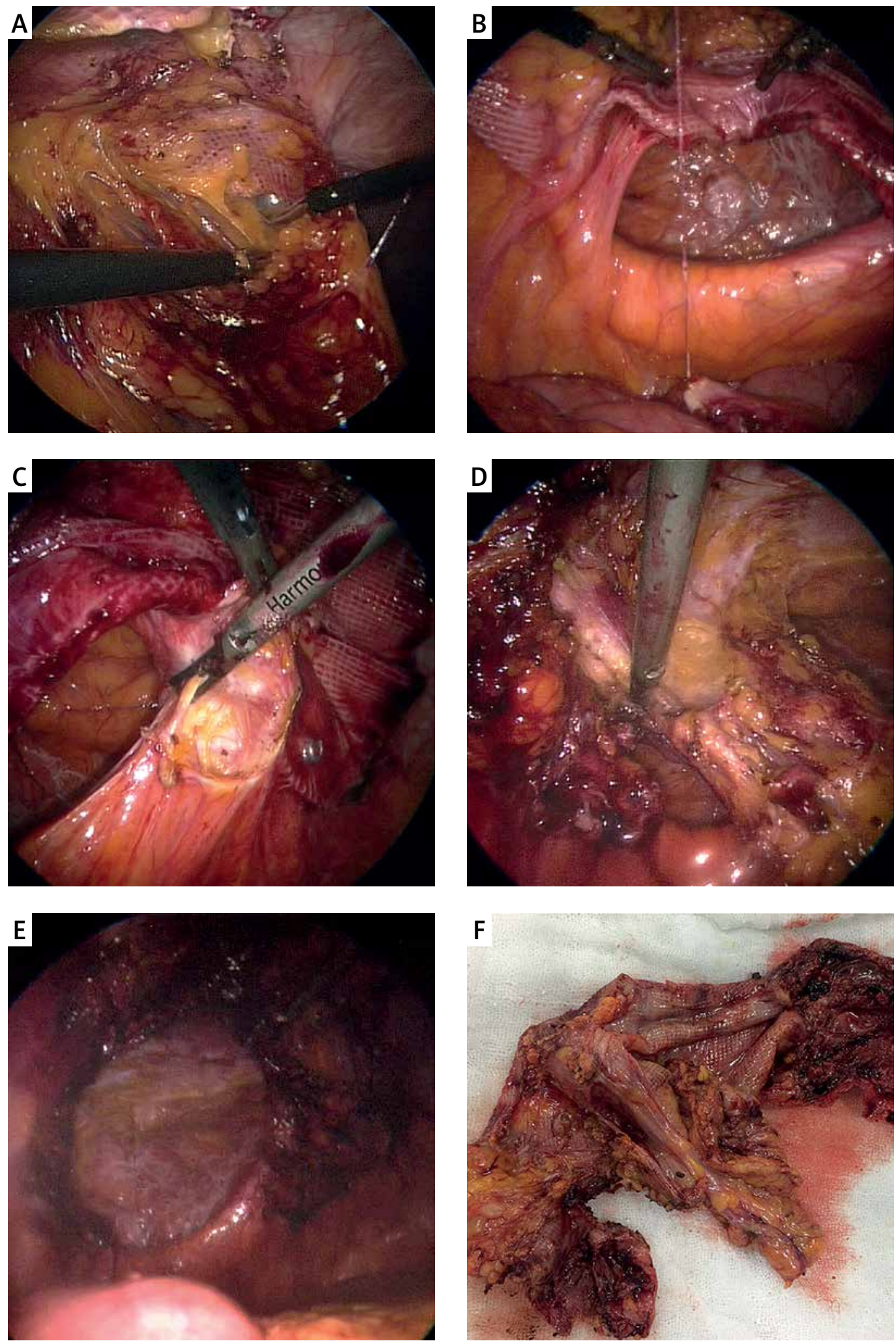

Photo 1. A - Adhesiolysis of adhesions to the mesh, B - recurrence and shrinkage of the mesh, C-excision of previous mesh and fixation materials, $\mathbf{D}$ - excision of the mesh from the hernia sac, $\mathbf{E}$ - appearance following removal of mesh, $\mathbf{F}$ - previous mesh following excision from the abdominal wall 
regarding NRS, on postoperative day 1 . Reduction of the NRS scores on remaining postoperative days was faster and NRS scores were lower on postoperative day 10 and at the $6^{\text {th }}$ week in the MR group $(p<0.05)$. Dissection of more planes during the excision of mesh could be responsible for the pain and higher NRS scores in the MR group on postoperative day 1. In addition, excision of the mesh and fixation materials could be associated with the significant decrease in pain on postoperative day 10 and at the sixth week. Patients in the MR group showed a similar pattern of pain to the patients who did not have a recurrent ventral hernia, differently from the non-MR group. Therefore, we compared the patients in the MR group with the patients who underwent laparoscopic ventral hernia repair for the first time (Table II). The shortest operative time was observed in primary incisional hernia repair patients and also mean hospital stay was shorter than the MR group $(p<0.05)$. Even though NRS score on postoperative day 1 was higher in the MR group, the scores were closer on the following days in comparison. Clinical appearance showed a similar pattern in both groups regarding pain. Comparison of the non-MR group and primary incisional hernia patients should also be mentioned. Although pain scores were higher in the non-MR group ( $p<0.05$ ), the groups were not comparable due to the characteristics of patients, and it represents the major limitation of our study due to the observational design. Further prospective comparative studies are required to enable this comparison.

Transfascial sutures have been associated with chronic pain previously. Four transfascial sutures were applied to all cases - except patients with suprapubic hernia - and the groups had a homogeneous distribution regarding the transfascial sutures. Therefore, we consider that findings of pain are not affected by transfascial sutures, in our series. We should mention that we were applying transfascial sutures for all cases to place the mesh properly due to large defects rather than permanent fixation, in the past. We do not prefer laparoscopic repair for hernias with a width larger than $8 \mathrm{~cm}$ at present and do not apply transfascial sutures - except suprapubic hernias. In addition, the complication of chronic seroma should be mentioned. Patients in the non-MR group had higher rates of chronic seroma than the MR group $(p<0.05)$. The number of patients was not appropriate to compare the number of aspirations. However, patients in the non-MR group had aspira- tions two or three times, whereas the MR group and primary incisional hernia patients had them once.

Closure of the defect is becoming more widely advocated nowadays [13-17]. We should mention that we were not closing the defects before applying mesh in the past, and we are closing the defects in selected cases now. Laparoscopic IPOM was the most preferred choice of treatment in our division, in the beginning. However, regarding the experience gained through the rising number of patients, we revised our preference through the requirement of patients [18]. Recurrent cases in our series had shown a larger width that pointed to a technical failure regarding our choice, in particular. Also, adhesions to the mesh regardless of previous abdominal surgery in the literature led us to limit the indications for laparoscopic IPOM repair [3]. Considering our methods at present, we decide on a repair according to the defect width; we prefer laparoscopic sublay repair for defects between 3 and $5 \mathrm{~cm}$, laparoscopic IPOM repair for defects between 5 and $8 \mathrm{~cm}$, and an open approach for defects larger than $8 \mathrm{~cm}$. We prefer to close the defects of patients with a single defect and do not close small multiple defects. Also, we prefer laparoscopic transperitoneal partial extraperitoneal repair, without closure of the defect, for suprapubic hernia regardless of defect width. The weak recommendation for closing the hernia defect in the most recent guideline supports our view in the past, by considering the studies based on authors' opinions rather than prospective comparative studies [19]. However, as a technical detail, closure of the defect for recurrent incisional hernias should be discussed. The patient in Photo 1 had a partially floating and contracted mesh with a partially covered defect which was possibly not suitable for a complete closure without mesh removal. Although hybrid methods were described previously, complete closure of the defect is impossible without removal of the mesh for most of the recurrent cases during laparoscopic hernia repair [13, 14]. Depending on technical improvements, such as the closure of the defect, mesh removal could be a preferred choice.

In the light of the present findings, removal of the previous mesh has shown the characteristics of a laparoscopic ventral hernia repair for the first time, regarding the pain. The observational design is the major limitation of our study. Long-lasting complaints of pain in the non-MR group could not be compared with the basal status of pain before sur- 
gery. Also, removal of the mesh was not suitable for all patients. The exact ratio of previous hernia repair methods is unknown, in our series. Patients in the MR group were selected during the surgery and did not have a homogeneous distribution - with a mesh partially floating inside the abdomen (possibly with IPOM repair previously) and did not have adherence to skin. Therefore, further prospective comparative studies are required to verify this opinion.

\section{Conclusions}

Mesh removal during laparoscopic repair of recurrent ventral hernias has an association with the reduction of pain. Long-lasting complaints of pain and complications such as a chronic seroma seemed to be associated with the existence of previous mesh. However, further prospective comparative studies are required to verify this view.

\section{Conflict of interest}

The authors declare no conflict of interest.

\section{References}

1. Muysoms FE, Antoniou SA, Bury K, et al. European Hernia Society guidelines on the closure of abdominal wall incisions. Hernia 2015; 19: 1-24.

2. Silecchia Giangranco, Campanile FC, Sanches L, et al. Laparoscopic ventral/incisional hernia repair: updated guidelines from the EAES and EHS endorsed Consensus Development Conference. Surg Endosc 2015; 29: 2463-84.

3. Sikar HE, Cetin K, Eyvaz K, et al. Laparoscopic repair of large suprapubic hernias. Videosurgery Miniinv 2017; 12: 245-50.

4. Sanchez LJ, Piccoli M, Ferrari CG, et al. Laparoscopic ventral hernia repair: results of a two thousand patients prospective multicentric database. Int J Surg 2018; 51: 31-8.

5. Sikar HE, Cetin K, Eyvaz K, et al. Lateral sided trocar site hernia following laparoscopic hernia repair: results of a long-term follow-up. Hernia 2019; 23: 101-6.

6. Sikar HE, Cetin K, Eyvaz K, et al. Evaluation of the effects of absorbable and nonabsorbable tacks on laparoscopic suprapubic hernia repair: a retrospective cohort study. Int I Surg 2019; 63: 16-21.

7. Kokotovic D, Bisgaard T, Helgstrand F. Long-term recurrence and complications associated with elective incisional hernia repair. JAMA 2016; 316: 1575-82.

8. Bueno-Lledo J, Torregrosa-Gallud A, Carreno-Saenz O, et al. Partial versus complete removal of the infected mesh after abdominal wall hernia repair. Am J Surg 2017; 214: 47-52.

9. Levy S, Moszkowicz D, Poghosyan T, et al. Comparison of complete versus partial mesh removal for the treatment of chronic mesh infection after abdominal wall hernia repair. Hernia 2018; 22: 773-9.
10. Ortega-Deballon P. Total or partial removal of infected mesh? Invited comment. Hernia 2018; 22: 951-2.

11. Sharma R, Fadaee N, Zarrinkhoo E, et al. Why we remove mesh. Hernia 2018; 22: 953-9.

12. Pandey H, Thakur DS, Somashekar U, et al. Use of polypropylene mesh in contaminated and dirty strangulated hernias: short-term results. Hernia 2018; 22: 1045-50.

13. Dey S, Parthasarathi R, Sabnis SC, et al. Laparoscopic management of recurrent ventral hernia: an experience of 222 patients. Hernia 2019; DOI: 10.1007/s10029-019-01912-0.

14. Ahonen-Siirtola M, Nevala T, Vironen J, et al. Laparoscopic versus hybrid approach for treatment of incisional ventral hernia: a prospective randomized multicentre study, 1-year results. Surg Endosc 2019 DOI: 10.1007/s00464-019-06735-9.

15. Tandon A, Pathak S, Lyons NJ, et al. Meta-analysis of closure of the fascial defect during laparoscopic incisional and ventral hernia repair. Br J Surg 2016; 103: 1598-607.

16. Baker JJ, Oberg S, Andresen K, et al. Decreased re-operation rate for recurrence after defect closure in laparoscopic ventral hernia repair with a permanent tack fixated mesh: a nationwide cohort study. Hernia 2018; 22: 577-84.

17. Mitura K, Skolimowska-Rzewuska M, Garnysz K. Outcomes of bridging versus mesh augmentation in laparoscopic repair of small and medium midline ventral hernias. Surg Endosc 2017; 31: 382-8.

18. Mitura K. Different approach to laparoscopic IPOM ventral hernia surgery - what has the last decade taught us? Pol Przegl Chir 2016; 88: 54-61.

19. Earle D, Roth JS, Saber A, et al. SAGES Guidelines Committee, SAGES guidelines for laparoscopic ventral hernia repair. Surg Endosc 2016; 30: 3163-83.

Received: 16.04.2019, accepted: 6.05.2019. 